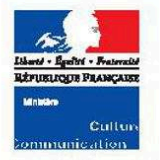

Secrétariat général

Délégation
au développen

et aux affaires

internationales

Département

des études,

de la prospective
et des statistiques

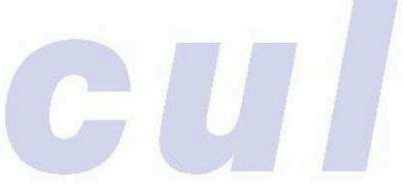

182, rue Saint-Honoré, 75033 Paris cedex 0

\title{
L'emploi
}

\section{dans les professions culturelles en 2005}

Éric Cléron, Frédérique Patureau* Cultural occupation employment 2005

Cette exploitation annuelle de l'Enquête Emploi de l'Insee ${ }^{1}$, réalisée par le DEPS, permet une estimation quantitative de l'emploi - et une caractérisation de cet emploi - dans les professions culturelles $^{2}$, c'est-à-dire dans les professions spécifiques du domaine des arts, des spectacles et de l'information.

À travers l'analyse des résultats 2005 , sont mis en évidence les grandes caractéristiques de ces emplois - en particulier le déséquilibre géographique au profit de l'Île-de-France, la part importante du non-salariat... - et le profil des personnes qui les occupent - leur haut niveau de diplôme tout particulièrement.

\section{Près DE $2 \%$}

\section{DE LA POPULATION ACTIVE OCCUPÉE}

En 2005, environ 457000 personnes exercent une profession culturelle, soit près de $2 \%$ de la population active occupée. Parmi ces actifs, un tiers appartient aux professions des arts plastiques et métiers d'art, $28 \%$ à celles de l'audiovisuel et du spectacle (voir graphique 1, p. 2).

Les professions littéraires, avec 58000 actifs, représentent $13 \%$ du total des professions culturelles, les professeurs d'art environ $11 \%$, et les professions d'architecte et de cadre et technicien de la documentation et de la conservation, entre 7 et $8 \%$ chacune.

\section{Avertissement au lecteur}

Les effectifs des professions culturelles étant relativement faibles, l'aléa introduit par l'échantillonnage de l'Enquête Emploi n'est pas toujours négligeable. II convient donc de considérer avec précaution les chiffres présentés ici. Toutefois, les tendances générales dégagées pour 2005 confirment celles qui ont été observées les années précédentes*.

Par ailleurs, le terme d' « actif́s " désigne en réalité ici les seuls " actif́s occupés " hors chômeurs.

Dans les graphiques, les chiffres donnés sont arrondis, mais la représentation graphique respecte la précision des données initiales.

"Voir http://wwww.culture.gouv.fr/deps publications statistiques: Notes de I'Observatore de l'emploi culturel, na 45, mai 2006.

\footnotetext{
* Respectivement attaché statisticien de l'Insee et ingénieur de recherche, chargés d'études au Département des études, de la prospective et des statistiques (Deps), coresponsables du programme «Dynamique des activités, de l'emploi et du travail ». L'exploitation et l'analyse ont été réalisées avec le concours d'Anaïs Le Gouguec.

1. Réalisée par l'Insee, l'Enquête Emplơi fournit des donnés sur les professions, l'activité des femmes ou des jeunes, la durée du travail, les emplois précaires et pemet de mieux cerner la situation des chômeurs et les changements de situation vis-à-vis du travail (voir www.insee.fr). 2. Voir définition dans l'encadré p. 6.
} 


\section{L'emploi dans les professions culturelles en 2005}

Cultural occupation employment 2005

\section{Eric Cléron et Frédérique Patureau}

Éditeur : Département des études, de la prospective et des statistiques Lieu d'édition : Paris

Année d'édition : 2007

Date de mise en ligne : 21 septembre 2015

Collection : Culture chiffres

ISBN électronique : 9782111398337

\section{Q books}

http://books.openedition.org

Édition imprimée

Nombre de pages : 8

Référence électronique

CLÉRON, Eric ; PATUREAU, Frédérique. L'emploi dans les professions culturelles en 2005. Nouvelle édition [en ligne]. Paris : Département des études, de la prospective et des statistiques, 2007 (généré le 25 avril 2021). Disponible sur Internet : <http://books.openedition.org/deps/797>. ISBN :

9782111398337

(C) Département des études, de la prospective et des statistiques, 2007

Creative Commons - Attribution - Pas d'Utilisation Commerciale 3.0 non transposé - CC BY-NC 3.0 

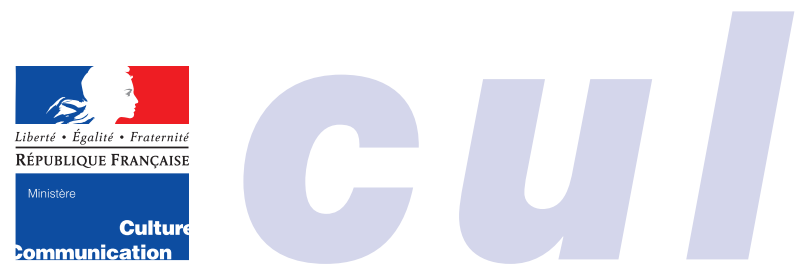

Secrétariat général

Délégation

au développement

et aux affaires

internationales

Département

des études,

de la prospective

et des statistiques

\section{L'emploi}

\section{dans les professions culturelles en 2005}

\section{Éric Cléron, Frédérique Patureau* Cultural occupation employment 2005}

Cette exploitation annuelle de l'Enquête Emploi de l'Insee ${ }^{1}$, réalisée par le DEPS, permet une estimation quantitative de l'emploi - et une caractérisation de cet emploi - dans les professions cultu-

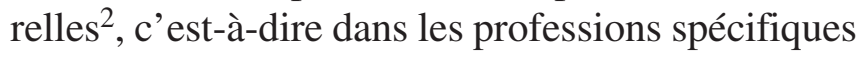
du domaine des arts, des spectacles et de l'information.

À travers l'analyse des résultats 2005, sont mis en évidence les grandes caractéristiques de ces emplois - en particulier le déséquilibre géographique au profit de l'île-de-France, la part importante du non-salariat... - et le profil des personnes qui les occupent - leur haut niveau de diplôme tout particulièrement.

\author{
PRÈS DE $2 \%$
}

\section{DE LA POPULATION ACTIVE OCCUPÉE}

En 2005, environ 457000 personnes exercent une profession culturelle, soit près de $2 \%$ de la population active occupée. Parmi ces actifs, un tiers appartient aux professions des arts plastiques et métiers d'art, $28 \%$ à celles de l'audiovisuel et du spectacle (voir graphique 1, p. 2).

Les professions littéraires, avec 58000 actifs, représentent $13 \%$ du total des professions culturelles, les professeurs d'art environ $11 \%$, et les professions d'architecte et de cadre et technicien de la documentation et de la conservation, entre 7 et $8 \%$ chacune.

\section{Avertissement au lecteur}

Les effectifs des professions culturelles étant relativement faibles, l'aléa introduit par l'échantillonnage de l'Enquête Emploi n'est pas toujours négligeable. II convient donc de considérer avec précaution les chiffres présentés ici. Toutefois, les tendances générales dégagées pour 2005 confirment celles qui ont été observées les années précédentes*.

Par ailleurs, le terme d' «actifs » désigne en réalité ici les seuls « actifs occupés » hors chômeurs.

Dans les graphiques, les chiffres donnés sont arrondis, mais la représentation graphique respecte la précision des données initiales.

*Voir http://www.culture.gouv.fr/deps publications statistiques: Notes de l'Observatoire de l'emploi culturel, n 45, mai 2006.

\footnotetext{
* Respectivement attaché statisticien de l'Insee et ingénieur de recherche, chargés d'études au Département des études, de la prospective et des statistiques (DEPS), coresponsables du programme «Dynamique des activités, de l'emploi et du travail ». L'exploitation et l'analyse ont été réalisées avec le concours d'Anaïs Le Gouguec.

1. Réalisée par l'Insee, l'Enquête Emploi fournit des données sur les professions, l'activité des femmes ou des jeunes, la durée du travail, les emplois précaires et permet de mieux cerner la situation des chômeurs et les changements de situation vis-à-vis du travail (voir www.insee.fr). 2. Voir définition dans l'encadré p. 6.
} 
1 - ... selon la profession

Professions de l'audiovisuel et du spectacle Artistes des spectacles

Professions des arts plastiques et des métiers d'art Artistes plasticiens Stylistes décorateurs Photographes

Professions littéraires Journalistes et cadres de l'édition

Auteurs littéraires, scénaristes, dialoguistes

Cadres et techniciens de la documentation et de la conservation

Professeurs d'art (hors établissements scolaires) Architectes

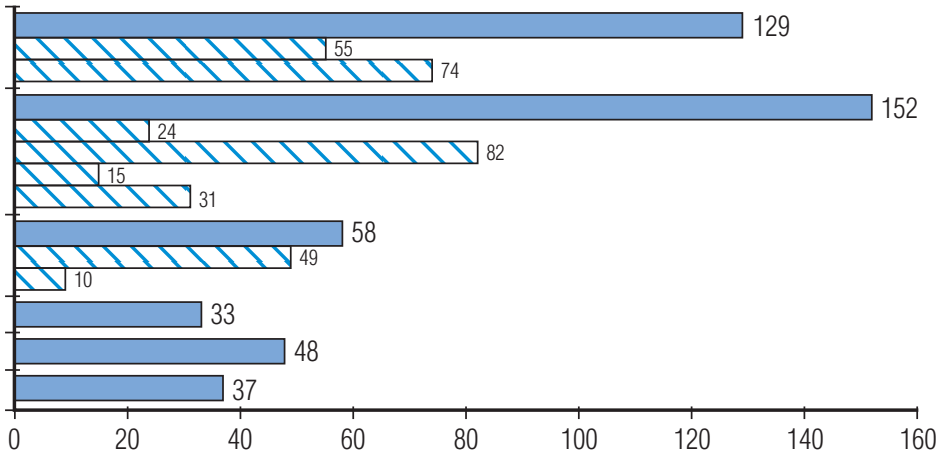

60 Milliers d'actifs

\section{2 - ... selon le pourcentage d'hommes par profession}

Ensemble de la population active occupée Professions culturelles Professions de l'audiovisuel et du spectacle Cadres, techniciens et ouvriers des spectacles Professions des arts plastiques et des métiers d'art Artistes plasticiens tylistes décorateurs Photographes
Métiers d'art

Professions littéraires Journalistes et cadres de l'édition Auteurs littéraires, scénaristes, dialoguistes

Cadres et techniciens de la documentation et de la conservation

Professeurs d'art (hors établissements scolaires) Architectes

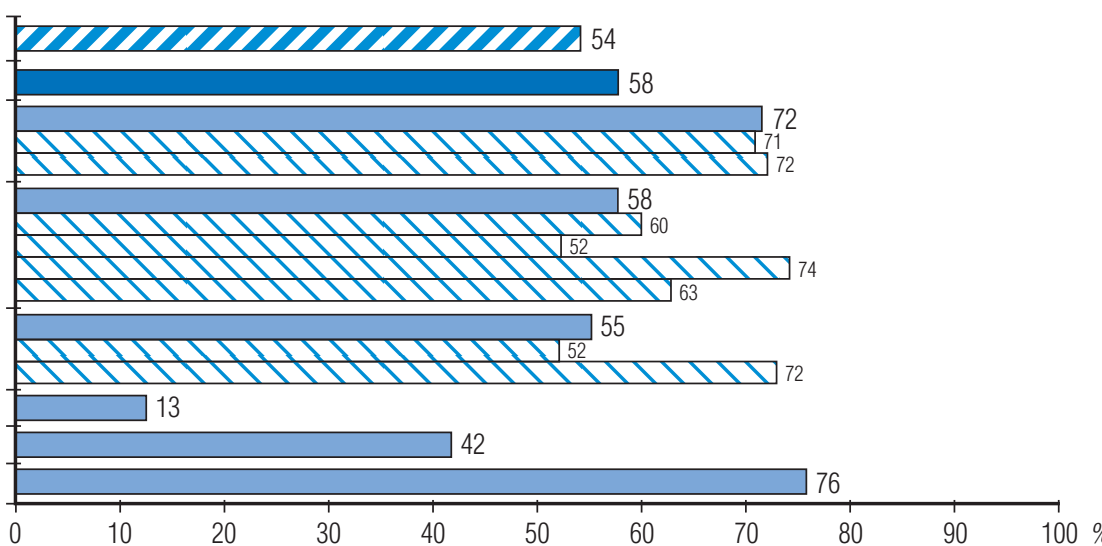

3 - ... selon la part des moins de 40 ans par profession

Ensemble de la population active occupée Professions culturelles Professions de l'audiovisuel et du spectacle Cadres, techniciens et ouvriers des spectacles Professions des arts plastiques et des métiers d'art Artistes plasticiens Photographes
Phón Photographes
Métiers d'art Professions littéraires Journalistes et cadres de l'édition Auteurs littéraires, scénaristes, dialoguistes

Cadres et techniciens de la documentation et de la conservation

Professeurs d'art (hors établissements scolaires) Architectes

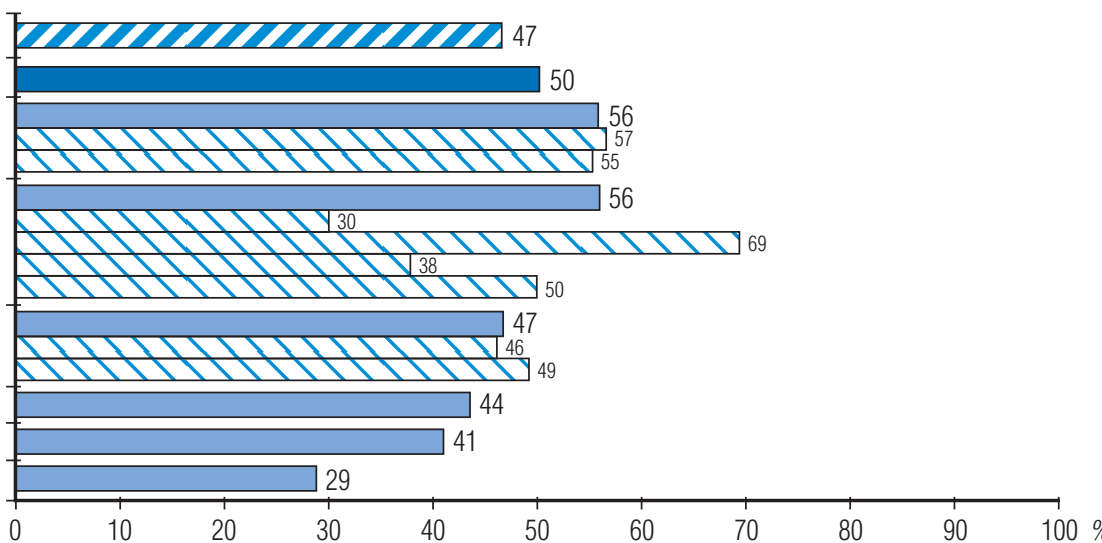

$4-\ldots$ selon la part des «bac +2 et plus » par profession

Ensemble de la population active occupée Professions culturelles

Professions de l'audiovisuel et du spectacle Cadres, techniciens et ouvriers des spectacles Professions des arts plastiques et des métiers d'art Artistes plasticiens tylistes décorateurs Photographes
Métiers d'art Professions littéraires Journalistes et cadres de l'édition Auteurs littéraires, scénaristes, dialoguistes

Cadres et techniciens de la documentation et de la conservation

Professeurs d'art (hors établissements scolaires)

Architectes
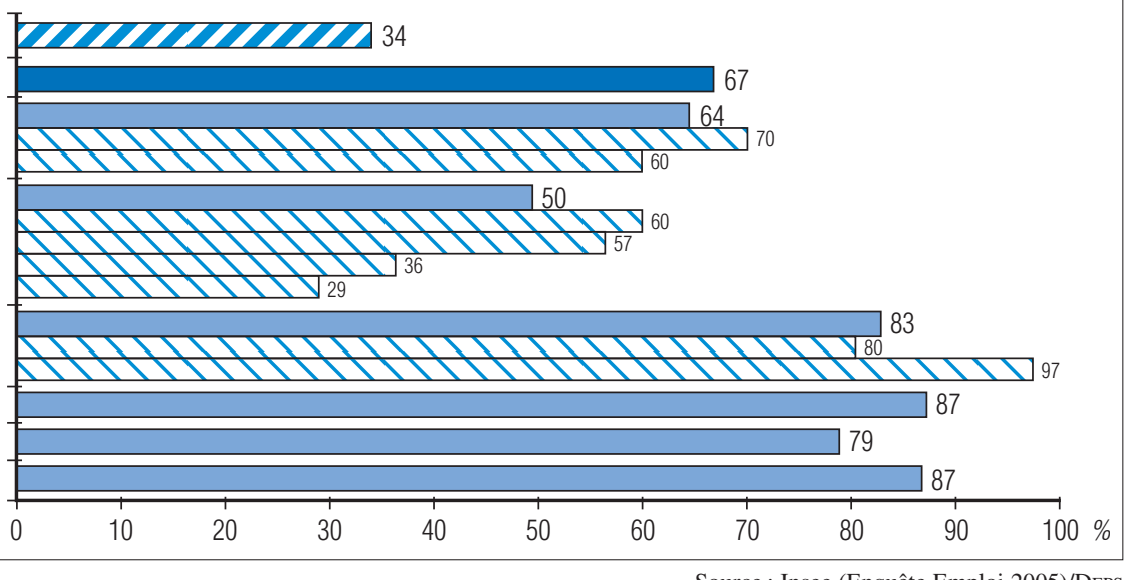


\section{UNE SURREPRÉSENTATION MASCULINE}

Si le phénomène de surreprésentation masculine est un peu plus marqué au sein des professions culturelles que dans l'ensemble de la population active occupée $(58 \%$ contre $54 \%$, voir graphique 2), il masque en fait de grandes disparités selon les professions considérées. Avec respectivement $76 \%, 74 \%$ et $73 \%$, les architectes, les photographes et les auteurs littéraires font partie des professions très nettement exercées par des hommes, alors que, à l'inverse, les effectifs féminins sont importants parmi les cadres et techniciens de la documentation et de la conservation ( $87 \%$ ) et chez les professeurs d'art (58\%).

\section{UN ACTIF SUR DEUX}

\section{A MOINS DE 40 ANS}

Les actifs des professions culturelles sont en moyenne un peu plus jeunes que ceux des autres professions (50\% de moins de 40 ans contre $47 \%$ dans l'ensemble de la population active occupée, voir graphique 3). La profession de styliste décorateur, avec $69 \%$ de moins de 40 ans, en fournit un exemple, comme l'ensemble des professions artistiques et techniques du spectacle (56\%).

D'autres professions, en revanche, sont exercées par des actifs en moyenne plus âgés : c'est le cas des architectes et des artistes plasticiens (où, respectivement, $29 \%$ et $30 \%$ des individus ont moins de 40 ans). Ces deux professions connaissent en outre un taux particulièrement élevé d'actifs de plus de 60 ans (entre $11 \%$ et $12 \%$, contre $2 \%$ pour l'ensemble des actifs occupés).

La répartition par âge est cependant plus homogène dans d'autres professions : c'est le cas notamment des auteurs littéraires et des professeurs d'art parmi lesquels la part des moins de 40 ans est proche de celle de l'ensemble des actifs ( $49 \%$ et $41 \%$ ), mais où les plus de 60 ans constituent pourtant une tranche relativement importante (entre $11 \%$ et $12 \%$ ).
UN NIVEAU ÉLEVÉ D'ÉTUDES GÉNÉRALES

Les professions culturelles, dans leur ensemble, se distinguent nettement de la moyenne de la population active en termes de niveau d'études générales : deux tiers des actifs ont un niveau supérieur ou équivalent à « bac $+2 »$ contre $34 \%$ pour l'ensemble de la population active occupée (voir graphique 4). Cette part atteint $97 \%$ chez les auteurs littéraires et $87 \%$ chez les architectes, mais n'est que de $36 \%$ chez les photographes et de $29 \%$ chez les professionnels des métiers d'art, ces derniers étant la seule profession culturelle dont la part des «bac +2 et plus » est inférieure à celle de l'ensemble de la population active occupée ${ }^{3}$.

\section{UNE CONCENTRATION DES ACTIFS SUR L'ÎLE-DE-FRANCE ET DANS LES GRANDES VILLES}

Avec 4 actifs sur 10 domiciliés en Île-de-France, les professions culturelles se distinguent fortement de l'ensemble de la population active occupée (19\%, voir graphique 5, p. 4). Mais, ici encore, cette situation recouvre en fait d'importantes disparités : le poids des actifs franciliens, s'il est de $60 \%$ pour les professions littéraires, est moins important dans d'autres domaines, jusqu'à devenir inférieur à la moyenne pour les métiers d'art, au sein desquels la répartition des actifs sur l'ensemble du territoire est plus homogène.

Ce déséquilibre géographique au profit de l'Îlede-France se double et se renforce, de façon plus générale, d'une concentration des actifs sur les zones urbaines: en effet, $78 \%$ des actifs des professions culturelles résident dans des communes appartenant à des pôles urbains ${ }^{4}$ - avec une concentration de $91 \%$ pour les auteurs littéraires -, proportion qui n'est que de $58 \%$ pour l'ensemble des actifs.

À la seule exception des métiers d'art, cette double concentration des actifs sur l'Île-de-France et les pôles urbains est remarquable dans chacune des catégories de professions culturelles.

3. Pour les photographes et les professionnels des métiers d'art, le niveau de compétence et de savoir-faire professionnels repose sur une formation spécifique souvent longue, mais largement en marge de la classification générale par niveau de diplôme inspirée des cursus universitaires.

4. Le pôle urbain est une unité urbaine offrant au moins 5000 emplois et qui n'est pas la couronne périurbaine d'un autre pôle urbain. 


\section{5 - ... selon le pourcentage de Franciliens par profession}

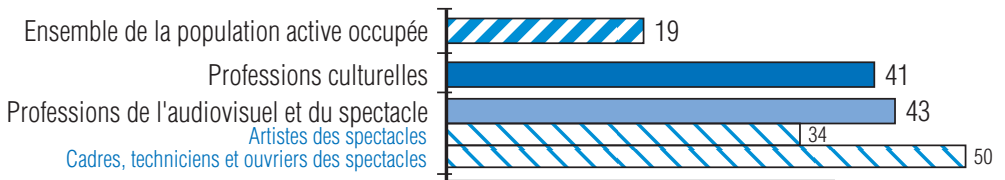
Cadres, techniciens et Artistes des spectacles Artistes plasticiens Stylistes décorateurs Photographes
Métiers d'art Professions littéraires

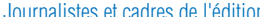
Auteurs littéraires, scénaristes, dialoguistes

Cadres et techniciens de la documentation et de la conservation

Professeurs d'art (hors établissements scolaires) Architectes
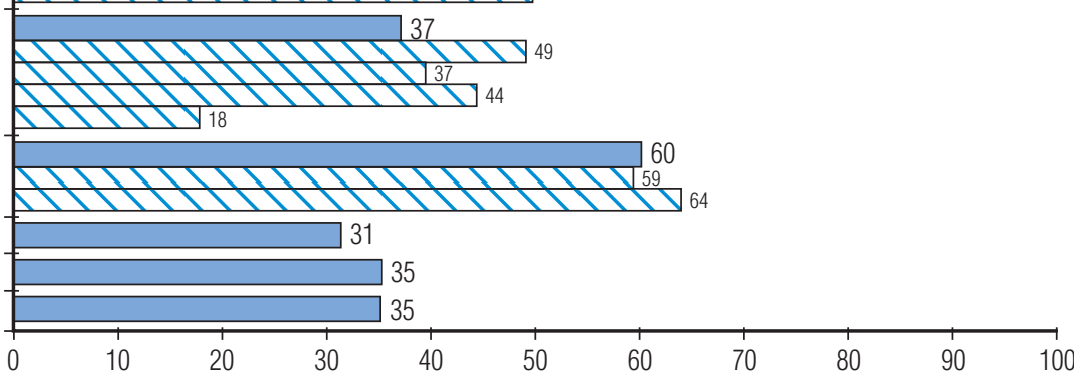

6 - ... selon la part de non-salariés par profession

Ensemble de la population active occupée

Professions culturelles

Professions de l'audiovisuel et du spectacle

Artistes des spectacles
Cadres, techniciens et ouvriers des spectacles

Professions des arts plastiques et des métiers d'art Artistes plasticiens

Stylistes décorateurs

Photographes

Professions littéraires Journalistes et cadres de l'édition

Auteurs littéraires, scénaristes, dialoguistes

Cadres et techniciens de la documentation et de la conservation

Professeurs d'art (hors établissements scolaires)

Architectes

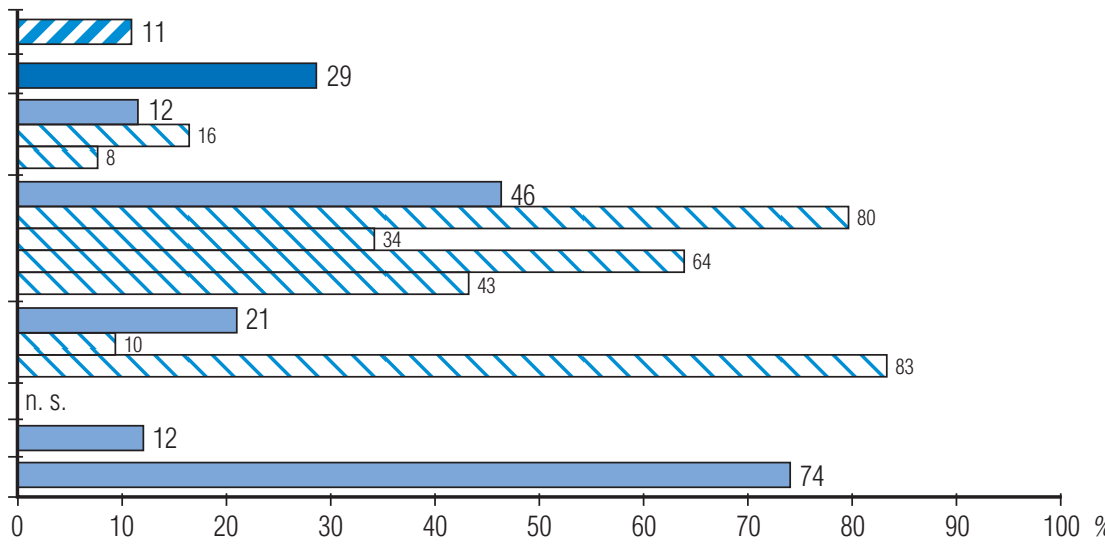

7 - ... selon la part des contrats à durée déterminée par profession

Ensemble de la population active occupée

Professions culturelles

Professions de l'audiovisuel et du spectacle

Artistes des spectacles
Cadres, techniciens et ouvriers des spectacles

Professions des arts plastiques et des métiers d'art Artistes plasticiens

Stylistes décorateurs

Photographes

Professions littéraires Journalistes et cadres de l'édition Journalistes et cadres de l'édition
Auteurs littéraires, scénaristes, dialoguistes Cadres et techniciens de la documentation et de la conservation

Professeurs d'art (hors établissements scolaires) Architectes

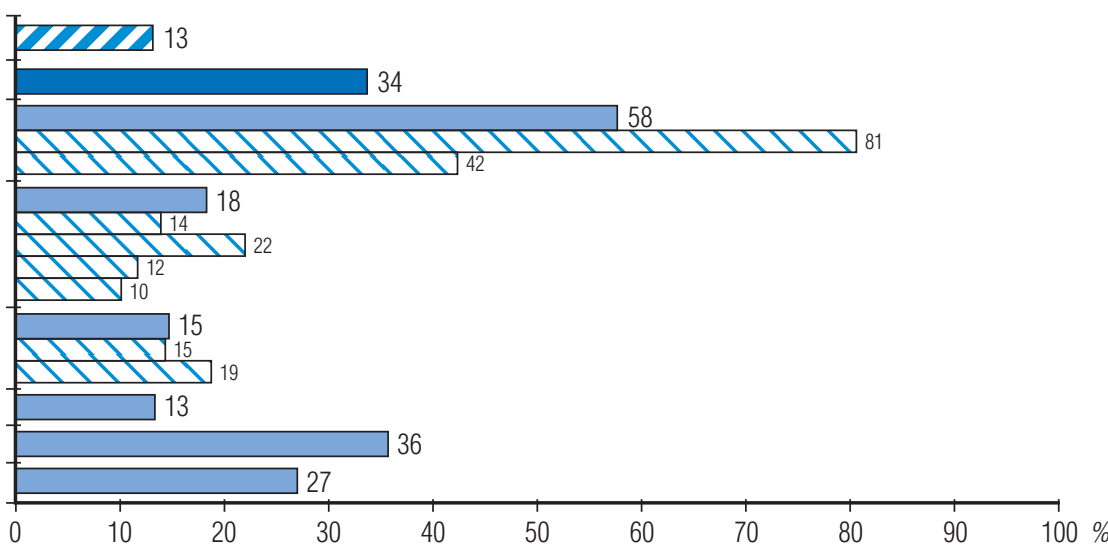

8 - ... selon la part des temps partiels par profession

Ensemble de la population active occupée

Professions culturelles

Professions de l'audiovisuel et du spectacle

Artistes des spectacles
Cadres, techniciens et ouvriers des spectacles

Professions des arts plastiques et des métiers d'art

Artistes plasticiens
Stylistes décorateurs

Photographes

Métiers d'art

Professions littéraires

Journalistes et cadres de l'édition

Auteurs littéraires, scénaristes, dialoguistes

Cadres et techniciens de la documentation et de la conservation

Professeurs d'art (hors établissements scolaires)

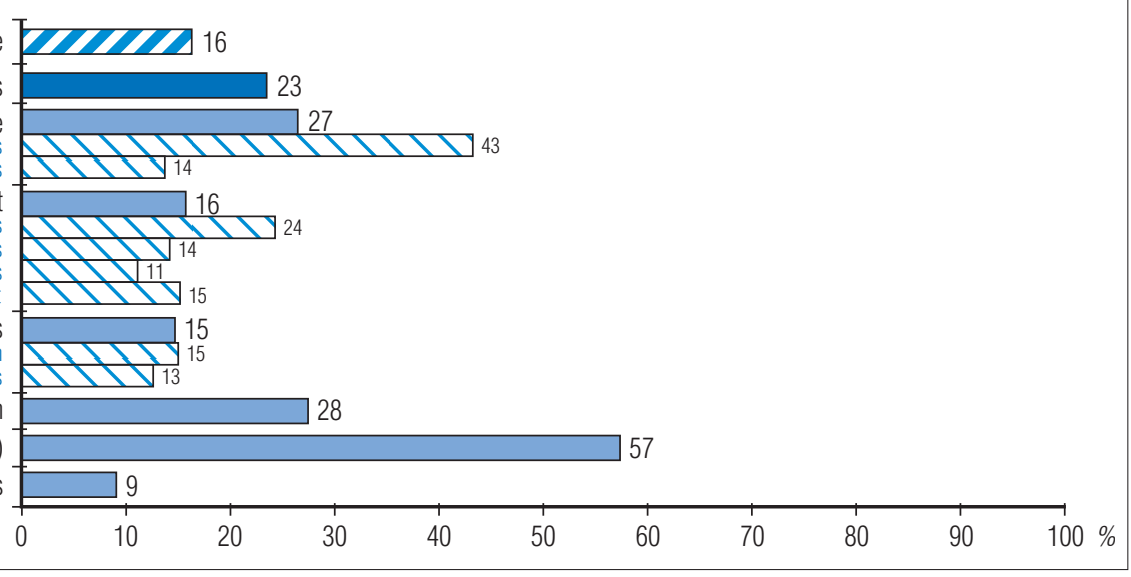

Architectes 


\section{Plus d'un actif sur Quatre N'EST PAS SALARIÉ}

La proportion des non-salariés parmi les professions culturelles est nettement plus élevée que dans l'ensemble des professions : $29 \%$ des actifs de ces professions travaillent à leur compte, ce qui n'est le cas que de $11 \%$ de la population active occupée (voir graphique 6).

Mais ici encore, les disparités sont grandes au sein des professions considérées. Le travail non salarié est très répandu parmi les auteurs littéraires (83\%), les artistes plasticiens (80\%), les architectes (74\%) ou encore les photographes (64\%). À l'inverse, le salariat est majoritairement présent chez les cadres et techniciens de la documentation et de la conservation (où le nombre de travailleurs indépendants est négligeable), chez les journalistes et les cadres de l'édition (90\%), les professeurs d'art $(88 \%)$ et parmi les professions de l'audiovisuel et du spectacle (88\% de salariés).

\section{UNE PRÉCARITÉ DE L'EMPLOI PLUS MARQUÉE QUE POUR L'ENSEMBLE DE LA POPULATION ACTIVE}

Comme le montrent plusieurs indicateurs - contrats à durée déterminée, emplois à temps partiel, situation de sous-emploi, exercice d'au moins une activité professionnelle secondaire à côté de l'activité déclarée comme principale ${ }^{5}-$, les conditions d'emploi sont plus précaires dans les professions culturelles que dans le reste de l'économie.

Ainsi, plus d'un salarié sur trois exerce sa profession dans le cadre d'un contrat à durée déterminée, contre $13 \%$ seulement dans l'ensemble des professions (voir graphique 7).

Cette caractéristique tient pour beaucoup aux professions artistiques et techniques de l'audiovisuel et du spectacle, où le recours à l'intermittence (donc au CDD dit d' «usage ») s'est généralisé au cours des vingt dernières années, avec des contrats de travail brefs, adaptés à la réalisation de projets artistiques ponctuels (réalisation d'un court ou d'un long-métrage, d'une émission de télévision, d'une mise en scène d'un spectacle, etc.). Ainsi en 2005, près des deux tiers des salariés de ces professions sont en CDD (et plus particulièrement, $80 \%$ des artistes de ces professions).

D'autres professions culturelles se caractérisent également par une proportion de CDD plus élevée, notamment celles de professeurs d'art (36\%).

Les actifs des professions culturelles sont également plus nombreux à travailler à temps partiel (23\% contre $16 \%$ de l'ensemble des actifs, voir graphique 8). C'est particulièrement le cas des professeurs d'art (57\%) et des artistes des spectacles $(43 \%)$.

Les architectes, à l'inverse, et quelques autres professions travaillent moins souvent à temps partiel que la population active occupée dans son ensemble (voir graphique 8).

Quant au fait d'exercer au moins une activité professionnelle secondaire, si c'est une pratique très peu répandue dans l'ensemble de la population active, elle est loin d'être négligeable pour les professeurs d'art, ainsi que, dans une moindre mesure, pour les artistes des spectacles (voir graphique 9, p. 6). Ce fait est probablement à mettre en relation avec l'importance des CDD dans ces deux secteurs, plusieurs contrats pouvant en effet être amenés à se chevaucher pour éviter d'éventuelles périodes chômées.

\section{UN DEGRÉ}

\section{DE « SATISFACTION » INÉGAL}

Le degré de satisfaction ${ }^{6}$ des actifs des professions culturelles vis-à-vis de leur profession est élevé même s'il est très légèrement inférieur à ce qu'il est pour l'ensemble de la population active (respectivement $73 \%$ et $78 \%$, voir graphique 10, p. 6).

On observe une part significative de «non-satisfaits » parmi les artistes des spectacles (46\%), les professeurs d'art et les photographes (respectivement $36 \%$ et $31 \%$ ). Ceci tient probablement au fait que ces professions sont fortement touchées par les

5. Ces activités professionnelles secondaires sont, dans l'Enquête Emploi, désignées par le terme d' «activités professionnelles supplémentaires ».

6. Un actif est déclaré «satisfait» s'il ne se trouve dans aucun de l'un des quatre cas suivants : souhait d'un autre emploi ; souhait d'effectuer un nombre d'heures de travail moins important (avec diminution correspondante de sa rémunération); souhait d'effectuer un nombre d'heures de travail plus important (avec augmentation correspondante de la rémunération) ou situation de sous-emploi. 
contrats à durée déterminée, le temps partiel, mais également par le sous-emploi ${ }^{7}$. En effet, les actifs des professions culturelles connaissent plus fréquemment des situations de sous-emploi que la moyenne de l'ensemble des actifs $(5 \%)$ : c'est le cas notamment des artistes des spectacles $(35 \%)$, des professeurs d'art $(26 \%)$ et des photographes (20\%) (voir graphique 10). À l'inverse, parmi les architectes et les auteurs littéraires, la proportion d'« insatisfaits » descend sous la barre des $16 \%$.

\section{Graphiques 9-10 - Répartition des actifs des professions culturelles...}

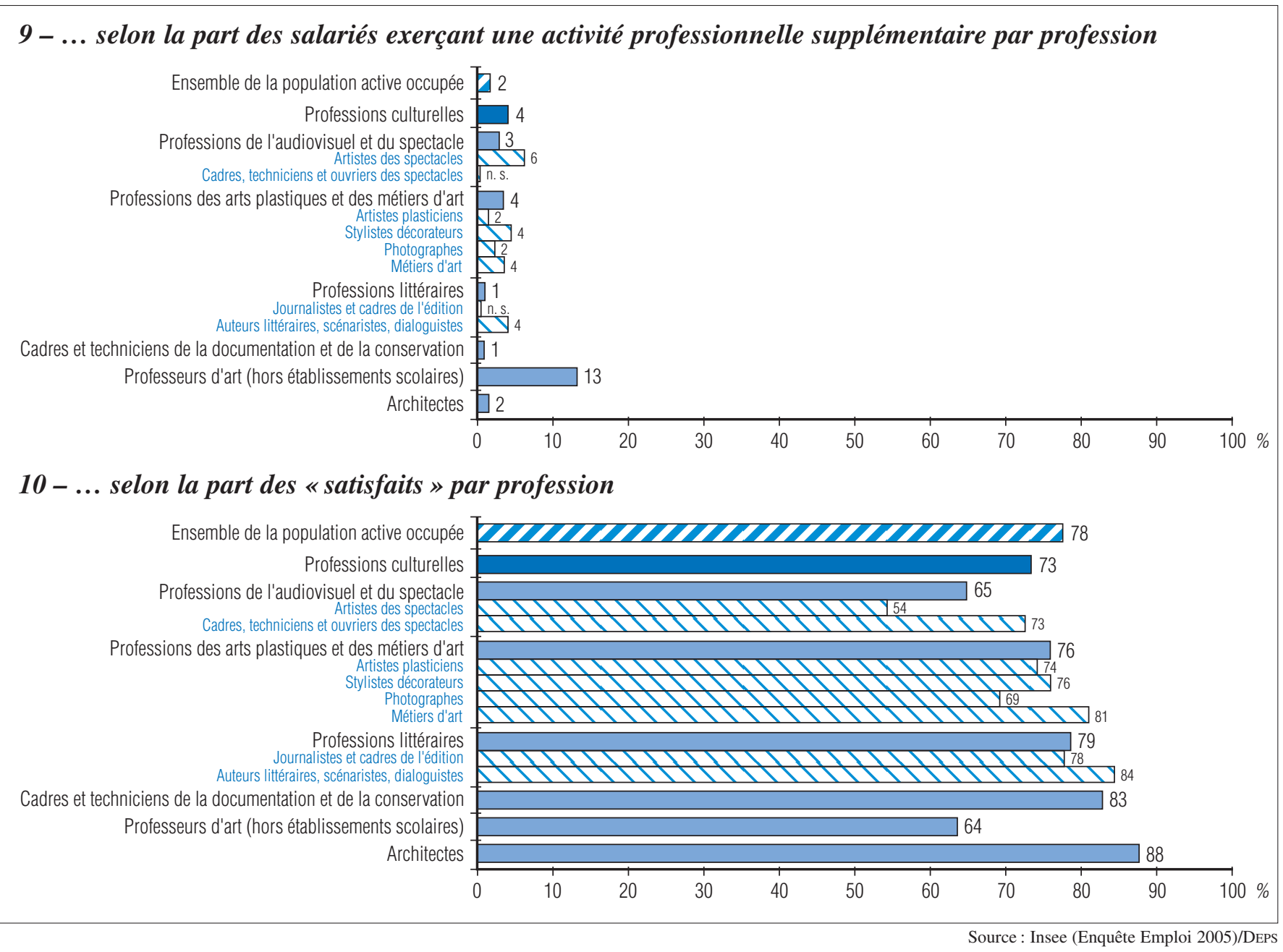

\section{Les professions culturelles : définition}

Les données présentées offrent une estimation quantitative des emplois, et leur caractérisation, dans les professions culturelles. Par «professions culturelles », il faut entendre "professions spécifiques de domaine des arts, des spectacles et de l'information ».

Cette approche par profession, définie par référence à la nomenclature nationale des professions et catégories socioprofessionnelles (Pcs), permet de décrire les caractéristiques des actifs des professions culturelles (en termes d'âge, de sexe, de niveau de diplôme...) et celles des emplois qu'ils occupent (nature du contrat de travail, salariat/non-salariat...), quel que soit le secteur d'activités (culturel ou non culturel) dans lequel ils travaillent. Toutes les professions, en effet, ne s'exercent pas dans des unités économiques du secteur culturel, c'est-àdire dans des unités dont l'activité principale, définie par référence à la nomenclature des activités française (NAF), relève du champ culturel : un designer, par exemple, peut exercer son emploi dans l'industrie automobile, un styliste dans l'industrie de l'habillement...

Comme chaque année, une autre note, établie à partir de la même Enquête Emploi, complète la présente approche, proposant une description des actifs et des emplois dans le secteur culturel, quelle que soit la nature de la profession qu'ils exercent (voir L'emploi dans le secteur culturel en 2005, Paris, Ministère de la culture et de la communication, DEPS, coll. « Culture chiffres » 2007-7).

http://www.culture.gouv.fr/deps publications statistiques

7. Un actif est en situation de sous-emploi s'il se trouve dans l'un des trois cas suivants : à temps partiel, recherchant un emploi pour travailler davantage; à temps partiel, ne recherchant pas un autre emploi, mais souhaitant travailler davantage et disponible; à temps complet, ayant involontairement travaillé moins que d'habitude (chômage partiel, chômage technique). 


\section{Le secteur culturel}

Liste des postes NAF

(Nomenclature des activités française) retenus pour sa définition

\section{INDUSTRIES CULTURELLES}

\section{Édition et librairie}

22.1A Édition de livres (livres, manuels scolaires, brochures, atlas, cartes et partitions musicales)

22.1G Édition d'enregistrement sonore (disques, disques compacts et bandes contenant de la musique ou d'autres enregistrements sonores)

22.1J Autres activités d'édition (calendriers, cartes postales, édition d'art: gravures, photographies, images)

52.4R Commerce de détail de livres, journaux et papeterie (et aussi fournitures de bureau et vente en kiosque)

\section{Presse}

22.1C Édition de journaux (quotidiens, journaux publicitaires)

22.1E Édition de revues et périodiques

92.4Z Agences de presse, journalistes indépendants

\section{Radio et télévision}

92.1A Production de films pour la télévision (films de tous types: séries, téléfilms...)

92.2A Activités de radio

92.2B Production de programmes de télévision

92.2D Édition de chaînes généralistes

92.2E Édition de chaînes thématiques

92.2F Distribution de bouquets de programmes de télévision

\section{Activités cinématographiques et de vidéo}

92.1B Production de films institutionnels et publicitaires (films publicitaires, films techniques d'entreprises, films de formation ou éducatifs, clips vidéo)

92.1C Production de films pour le cinéma (courts ou longs métrages destinés à la projection en salle)

92.1D Prestations techniques pour le cinéma et la télévision (prise de son, effets spéciaux, doublages...)

92.1F Distribution de films cinématographiques (vente ou location de films ou vidéos à d'autres établissements)

92.1G Édition et distribution vidéo (vente ou location de films ou vidéos à destination du public)

92.1J Projection de films cinématographiques

\section{ARCHITECTURE}

74.2A Activités d'architecture (projets architecturaux, conseils aux maîtres d'ouvrage, conduite d'opérations pour le compte de propriétaires, conseils et études en matière d'aménagement urbain et paysager)

\section{SPECTACLE VIVANT ET ACTIVITÉS ARTISTIQUES}

92.3A Activités artistiques (artistes indépendants: acteurs, musiciens, danseurs; ensembles permanents : orchestres, troupes, compagnies; créations de spectacles; et les autres artistes indépendants: peintres, dessinateurs, sculpteurs, écrivains...); organisation de manifestations culturelles, promotion

92.3B Services annexes aux spectacles (machinerie, costumes, maquillage, éclairage)

92.3D Gestion de salles de spectacles (salles de concerts, théâtres, cabarets, studios d'enregistrement)

92.3K Activités diverses du spectacle (cirque, marionnettes, son et lumière, clubs de danse, organisation de bals et de soirées sans exploitation d'un lieu particulier)

\section{CONSERVATION DU PATRIMOINE}

92.5A Gestion des bibliothèques

92.5C Gestion du patrimoine culturel (musées, sites, monuments historiques)

\section{Les professions culturelles}

Liste des postes Pcs (professions et catégories socioprofessionnelles) retenus pour sa définition

\section{PROFESSIONS DE L'AUDIOVISUEL ET DU SPECTACLE}

\section{Artistes des spectacles}

354b Artistes de la musique et du chant

354c Artistes dramatiques

354d Artistes de la danse, du cirque et des spectacles divers

\section{Cadres, techniciens et ouvriers des spectacles}

353b Directeurs, responsables de programmation et de production de l'audiovisuel et des spectacles

$353 c$ Cadres artistiques et technico-artistiques de la réalisation de l'audiovisuel et des spectacles

$465 \mathrm{~b}$ Assistants techniques de la réalisation des spectacles vivants et audiovisuels (salariés ou indépendants)

637c Ouvriers et techniciens des spectacles vivants et de l'audiovisuel

227a Indépendants gestionnaires de spectacle ou de service récréatif

\section{PROFESSIONS DES ARTS PLASTIQUES ET DES MÉTIERS D'ART}

\section{Artistes plasticiens}

354a Artistes plasticiens

\section{Stylistes décorateurs}

465a Concepteurs et assistants techniques des arts graphiques, de la mode et de la décoration (indépendants et salariés)

\section{Photographes}

465c Photographes (salariés et indépendants)

\section{Métiers d'art}

214e Artisans d'art

637b Ouvriers d'art

\section{PROFESSIONS LITTÉRAIRES}

\section{Journalistes et cadres de l'édition}

352a Journalistes et rédacteurs en chef

353a Directeurs de journaux, administrateurs de presse, directeurs d'édition (littéraire, musicale, audiovisuelle, multimédia)

\section{Auteurs littéraires}

352b Auteurs littéraires, scénaristes, dialoguistes

\section{CADRES ET TECHNICIENS DE LA DOCUMENTATION}

\section{ET DE LA CONSERVATION}

351a Bibliothécaires, archivistes, conservateurs, de la fonction publique

$372 f$ Cadres de la documentation, de l'archivage (hors fonction publique)

425a Assistants techniques de la documentation, de l'archivage (hors fonction publique) (dont les bibliothécaires adjoints, documentalistes)

PROFESSEURS D'ART (hors établissements scolaires) $354 \mathrm{~g}$ Professeurs d'art (hors établissements scolaires)

\section{ARCHITECTES}

$312 f$ Architectes libéraux

382b Architectes salariés 
Questions
de culture

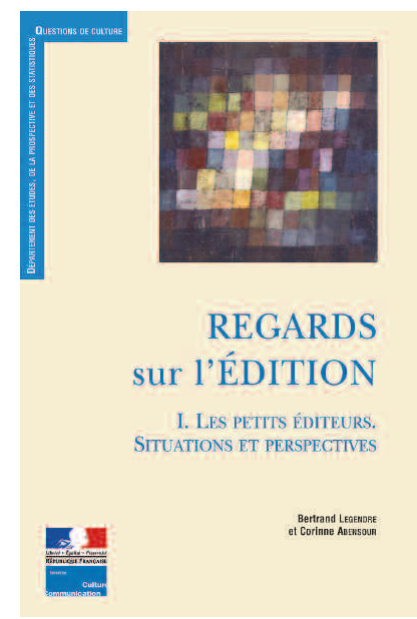

\section{Chiffres clés}
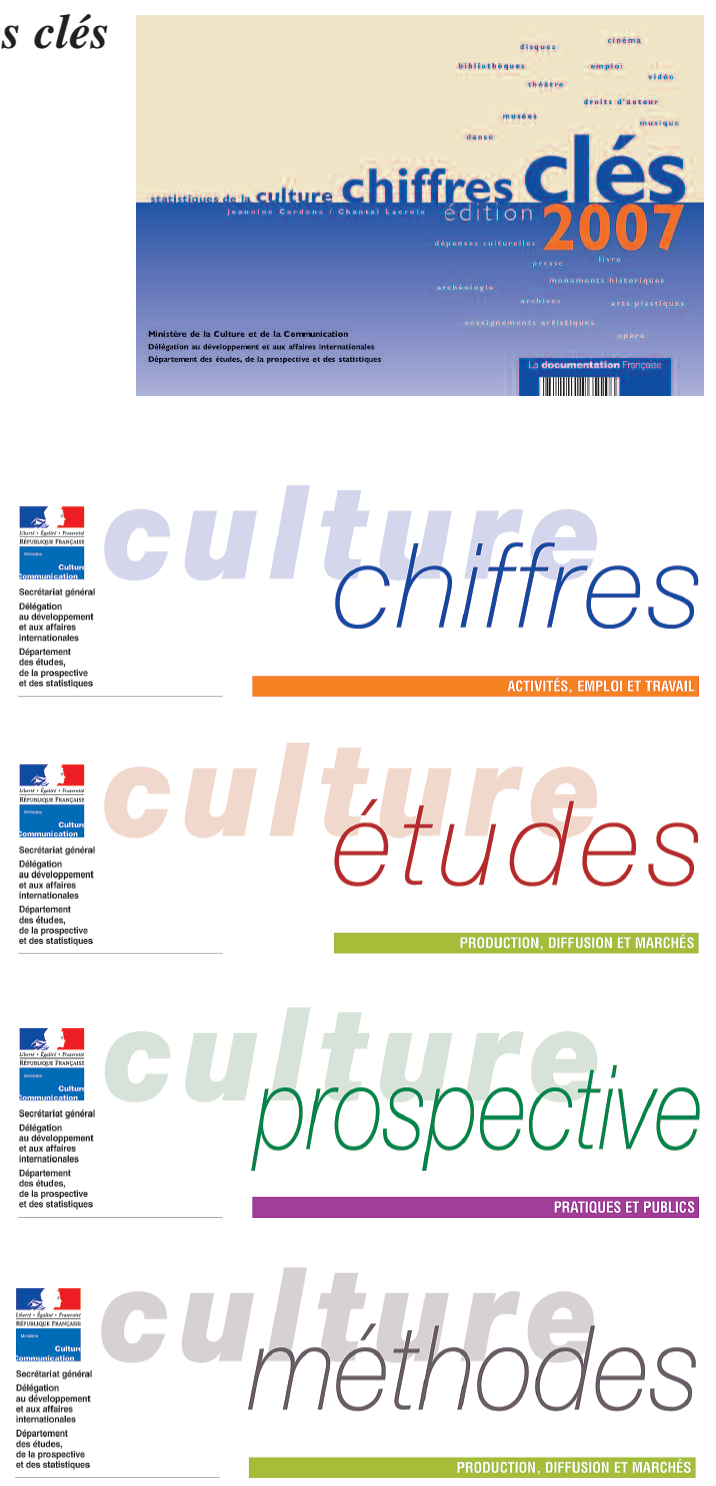

La collection «Questions de culture » (diffusée par la Documentation française) présente une analyse des résultats de travaux d'études ou de recherches en sciences économiques et sociales exécutés dans le cadre du programme du DEPS. Ils visent à rendre disponibles auprès du grand public des résultats ou des approches fermement établis sur les phénomènes culturels mais aussi des recherches innovantes où hypothèses nouvelles et rigueur scientifique s'allient pour penser ces évolutions.

L'annuaire statistique Chiffres clés* présente sous forme de tableaux et graphiques les statistiques relatives à la vie culturelle en France. L'étude aborde le spectre très large du champ culturel (patrimoine, spectacle, enseignements artistiques...) et propose une approche transversale sur les grands thèmes de politique culturelle (emploi, financement, pratiques). L'édition 2007 est publiée sous deux formes: imprimée (en vente à la Documentation française) et téléchargeable sur le site*.

Fidèle à sa vocation de service statistique ministériel, le DEPs présente dans cette collection les résultats chiffrés, les informations statistiques issues de ses travaux. Cette nouvelle collection prend la suite des anciennes (Notes de l'observatoire de l'emploi culturel, Notes statistiques)*.

Cette collection présente les synthèses des études et recherches réalisées par le département, publiées ou non dans la collection «Questions de culture ». Elle succède ainsi à Développement culturel*.

Consacrée à l'investigation d'hypothèses, à des mises en relation originales et à des travaux de prospective sur des champs connus comme sur des terrains plus expérimentaux, cette collection est ouverte à des travaux réalisés au DEPs mais également à des travaux extérieurs (recherches, thèses, etc.) afin de susciter le débat et les échanges au sein de la communauté scientifique*.

Cette collection met à la disposition du public des notes de méthodes dans les domaines qui relèvent des compétences du département (économie, sociologie, statistiques, etc.)*.

\section{* Ces collections sont téléchargeables sur www.culture.gouv.fr/deps rubrique "publications "}

Le DEPS n'assurant pas de diffusion physique de ces documents, nous vous proposons de vous informer régulièrement des parutions par message électronique.

Pour ce faire, merci de bien vouloir nous communiquer votre courriel à l'adresse

contactdeps.ddai@culture.gouv.fr

en indiquant comme sujet du message : "diffusion des collections du Deps". 